PROCEEDINGS OF THE

AMERICAN MATHEMATICAL SOCIETY

Volume 131, Number 6, Pages 1931-1933

S 0002-9939(02)06738-2

Article electronically published on September 19, 2002

\title{
MEANS ON SOLENOIDS
}

\author{
PAWEŁ KRUPSKI
}

(Communicated by Alan Dow)

Abstract. It is determined which solenoids admit 2-argument continuous means.

A mean on a topological space $X$ is a continuous mapping $m: X \times X \rightarrow X$ satisfying $m(x, y)=m(y, x)$ and $m(x, x)=x$, for every $x, y \in X$.

There is quite a large literature concerning means, and the main problem is which spaces admit means (see, e.g., [1], [2], [3], [4], [5], [6], [7] where further references are given).

Let $n_{1}, n_{2}, \ldots$ be a sequence of integers greater than 1 . A solenoid $\Sigma\left(n_{1}, n_{2}, \ldots\right)$ is the inverse limit $\lim _{(}\left(S_{k}, f_{k}\right)$, where $S_{k}=\{z \in \mathbb{C}:|z|=1\}$ and $f_{k}: S_{k+1} \rightarrow S_{k}$ is given by $f_{k}(z)=z^{n_{k}}$. Each solenoid is an abelian topological group under the coordinatewise multiplication $\left(z_{1}, z_{2}, \ldots\right)\left(z_{1}^{\prime}, z_{2}^{\prime}, \ldots\right)=\left(z_{1} z_{1}^{\prime}, z_{2} z_{2}^{\prime}, \ldots\right)$ with the neutral element $\mathbf{1}=(1,1, \ldots)$. Topologically, solenoids are indecomposable continua whose proper nondegenerate subcontinua are arcs.

Much is known about the existence of homomorphic means on topological abelian groups (see, e.g. 4]); in particular, a mean

$$
m: \Sigma\left(n_{1}, n_{2}, \ldots\right) \times \Sigma\left(n_{1}, n_{2}, \ldots\right) \rightarrow \Sigma\left(n_{1}, n_{2}, \ldots\right)
$$

which is a group homomorphism exists (and is unique) if and only if

$$
\text { there are infinitely many even numbers in the sequence } n_{1}, n_{2}, \ldots
$$

For example, if all numbers in the sequence are of the form $n_{i}=2 k_{i}, i \in \mathbb{N}$, then the formula

$$
m\left(\left(z_{1}, z_{2}, \ldots\right),\left(z_{1}^{\prime}, z_{2}^{\prime}, \ldots\right)\right)=\left(\left(z_{2} z_{2}^{\prime}\right)^{k_{1}},\left(z_{3} z_{3}^{\prime}\right)^{k_{2}}, \ldots\right)
$$

defines a homomorphic mean on $\Sigma\left(n_{1}, n_{2}, \ldots\right)$ (see [4]).

The question of whether there exist (non-homomorphic) means on solenoids for odd numbers in the sequence has been asked by J. J. Charatonik at seminars and by A. Illanes at the Spring Topology and Dynamical Systems Conference in 2001.

In this note we completely answer the question by showing that the same criterion (1) is necessary and sufficient for the existence of a mean on $\Sigma\left(n_{1}, n_{2}, \ldots\right)$.

Received by the editors April 2, 2001 and, in revised form, January 14, 2002.

2000 Mathematics Subject Classification. Primary 54H11, 54F15.

Key words and phrases. Mean, solenoid.

The paper was written during the author's visit to the Instituto de Matemáticas, Universidad Nacional Autónoma de México in 2000-2001. 
A simple proof is based on a method of [4 and the following proposition due to W. Scheffer [8, Corollary 2, p. 566].

Proposition 1. If $A$ and $B$ are continua which are topological groups with neutral elements $e_{A}$ and $e_{B}$, respectively, $B$ is abelian and $f: A \rightarrow B$ is a continuous mapping such that $f\left(e_{A}\right)=e_{B}$, then there exists a (unique) continuous homomorphism $h: A \rightarrow B$ which is homotopic to $f$.

Theorem 2. A solenoid $\Sigma\left(n_{1}, n_{2}, \ldots\right)$ admits a mean if and only if condition (1) is satisfied.

Proof. If there are infinitely many even numbers in the sequence $n_{1}, n_{2}, \ldots$, then $\Sigma\left(n_{1}, n_{2}, \ldots\right)$ is homeomorphic to $\Sigma\left(n_{1}^{\prime}, n_{2}^{\prime}, \ldots\right)$, where all numbers $n_{1}^{\prime}, n_{2}^{\prime}, \ldots$ are even. So we can assume that numbers $n_{1}, n_{2}, \ldots$ are even and define a mean by formula (2).

Conversely, let $m$ be a mean on $\Sigma\left(n_{1}, n_{2}, \ldots\right)$ and suppose there exists a number $k$ such that if $i \geq k$, then $n_{i}$ is odd. Then $\Sigma\left(n_{1}, n_{2}, \ldots\right)$ is homeomorphic to $\Sigma\left(n_{k}, n_{k+1}, \ldots\right)$, hence we can assume that all $n_{1}, n_{2}, \ldots$ are odd. Since $m(\mathbf{1}, \mathbf{1})=$ $\mathbf{1}$, there exists a continuous homomorphism

$$
h: \Sigma\left(n_{1}, n_{2}, \ldots\right) \times \Sigma\left(n_{1}, n_{2}, \ldots\right) \rightarrow \Sigma\left(n_{1}, n_{2}, \ldots\right)
$$

which is homotopic to $m$ by Proposition 1 We use the symbol $\sim$ to indicate that the values of homotopic mappings lie in the same arc component of $\Sigma\left(n_{1}, n_{2}, \ldots\right)$.

For every $\mathbf{z} \in \Sigma\left(n_{1}, n_{2}, \ldots\right)$, put

$$
\mu(\mathbf{z})=m(\mathbf{z}, \mathbf{1})=m(\mathbf{1}, \mathbf{z}) .
$$

We have

$$
\mu\left(\mathbf{z z}^{\prime}\right) \sim h\left(\mathbf{z z}^{\prime}, \mathbf{1}\right)=h(\mathbf{z}, \mathbf{1}) h\left(\mathbf{z}^{\prime}, \mathbf{1}\right) \sim \mu(\mathbf{z}) \mu\left(\mathbf{z}^{\prime}\right)
$$

which implies that

$$
\mathbf{z} \sim h(\mathbf{z}, \mathbf{z})=h(\mathbf{z}, \mathbf{1}) h(\mathbf{1}, \mathbf{z}) \sim \mu(\mathbf{z})^{2} \sim \mu\left(\mathbf{z}^{2}\right) .
$$

Now, if $\mathbf{z}=(-1,-1, \ldots) \in \Sigma\left(n_{1}, n_{2}, \ldots\right)$, then, by (3), $\mathbf{z} \sim \mu\left(\mathbf{z}^{2}\right)=\mu(\mathbf{1})=\mathbf{1}$; hence both points $\mathbf{z}$ and $\mathbf{1}$ lie in the same arc component of $\Sigma\left(n_{1}, n_{2}, \ldots\right)$ but this is not the case.

The author is grateful to W. J. Charatonik for his remarks.

\section{REFERENCES}

1. P. Bacon, Unicoherence in means, Colloq. Math. 21 (1970), 211-215. MR 41:9230

2. J. J. Charatonik, Some problems concerning means on topological spaces, Topology, Measures and Fractals (C. Bandt, J. Flachsmayer and H. Haase, eds.), Mathematical Research, vol. 66, Akademie Verlag, Berlin 1992, 166-177. MR 94d:54043

3. J. J. Charatonik, W. J. Charatonik, K. Omiljanowski and J. R. Prajs, Hyperspace retractions for curves, Dissertationes Math. (Rozprawy Mat.) 370 (1997), 1-34. MR 98j:54061

4. B. Eckmann, Räume mit Mittelbildungen, Comment. Math. Helv. 28 (1954), 329-340. MR 16:503g

5. A. Illanes, The Buckethandle continuum admits no mean, preprint.

6. A. Illanes and S. B. Nadler, Jr., Hyperspaces, Marcel Dekker, Inc., New York 1999. MR 99m:54006 
7. K. Kawamura and E. D. Tymchatyn, Continua which admit no mean, Colloq. Math. 71 (1996), 97-105. MR 97i:54045

8. W. Scheffer, Maps between topological groups that are homotopic to homomorphisms, Proc. Amer. Math. Soc. 33 (1972), 562-567. MR 46:288

Mathematical Institute, University of Wrockaw, Pl. Grunwaldzki 2/4, 50-384 WrocŁaw, Poland - and - Instituto de Matemáticas, UnAm, Circuito Exterior, Ciudad Universitaria, 04510 MÉxico, D. F., MÉXico

E-mail address: krupski@math.uni.wroc.pl 